\title{
Treatment Strategies Targeting Excess Hippocampal Activity Benefit Aged Rats with Cognitive Impairment
}

\author{
Ming Teng Koh*,', Rebecca P Haberman', Stacey Foti', Thomas J McCown ${ }^{2,3}$ and Michela Gallagher' \\ 'Department of Psychological and Brain Sciences, Johns Hopkins University, Baltimore, MD, USA; ${ }^{2}$ Gene Therapy Center, University of North \\ Carolina at Chapel Hill, NC, USA; ${ }^{3}$ Department of Psychiatry, University of North Carolina at Chapel Hill, NC, USA
}

\begin{abstract}
Excess neural activity in the CA3 region of the hippocampus has been linked to memory impairment in aged rats. We tested whether interventions aimed at reducing this excess activity would improve memory performance. Aged (24 to 28 months old) male Long-Evans rats were characterized in a spatial memory task known to depend on the functional integrity of the hippocampus, such that aged rats with identified memory impairment were used in a series of experiments. Overexpression of the inhibitory neuropeptide $Y$ I3-36 in the CA3 via adeno-associated viral transduction was found to improve hippocampal-dependent long-term memory in aged rats, which had been characterized with impairment. Subsequent experiments with two commonly used antiepileptic agents, sodium valproate and levetiracetam, similarly produced dose-dependent memory improvement in such aged rats. Improved spatial memory with low doses of these agents was observed in both appetitve and aversive spatial tasks. The benefits of these different modalities of treatment are consistent with the concept that excess activity in the CA3 region of the hippocampus is a dysfunctional condition that may have a key role underlying age-related impairment in hippocampal-dependent memory processes. Because increased hippocampal activation occurs in age-related memory impairment in humans as observed in functional neuroimaging, the current findings also suggest that low doses of certain antiepileptic drugs in cognitively impaired elderly humans may have therapeutic potential and point to novel targets for this indication.

Neuropsychopharmacology (2010) 35, I016-1025; doi:10.1038/npp.2009.207; published online 23 December 2009
\end{abstract}

Keywords: neurocognitive aging; neural hyperactivity; water maze; radial arm maze; mild cognitive impairment; NPY

\section{INTRODUCTION}

Aging is often associated with a decline in memory functions that depend on the hippocampus. Recent research on neurocognitive aging has focused on localized increased neural activity that may drive dysfunction underlying memory impairment in the hippocampal network (Wilson et al, 2006). Excess neural activity in the CA3 region of the hippocampus occurs in aged rats with memory impairment when such neurons are unable to encode new information in a manner usually observed in young rats (Wilson et al, 2003, 2005). The current research was designed to test the functional significance of such excess activity on behavioral performance in hippocampal-dependent spatial memory tasks.

Specialized functions of the subregions of the hippocampus have recently elucidated a critical role for the CA3/ dentate gyrus in the encoding of information. When young

*Correspondence: Dr MT Koh, Department of Psychological and Brain Sciences, Johns Hopkins University, Ames Hall, 3400, North Charles Street, Baltimore, MD 21218, USA, Tel: + 14105167724 Fax: + | 410516 0494, E-mail: mtkoh@jhu.edu

Received 30 July 2009; revised 16 October 2009; accepted 19 November 2009 rats first explore a familiar arena and then are placed in a novel environment, CA3 cells with location-specific encoding (eg, place fields) rapidly form different patterns of spatial encoding for the two environments (Leutgeb et al, 2004, 2007). In aged rats, on the other hand, hippocampal CA3 neurons can have very similar spatial representations across environments (Wilson et al, 2003, 2005). Hence, rather than creating a new representation for a novel environment, aged rats tend to retrieve a previously stored representation, a process known as 'pattern completion', distinguishing it from 'pattern separation'. Furthermore, the extent of this failure to encode new information predicts the degree of spatial learning impairment (Wilson et al, 2003). This positive correlation between behavior and physiology supports the idea that the encoding failure of hippocampal CA3 neurons contributes to impaired performance in settings that depend on spatial memory. The concurrent localization of excess activity in the CA3 population of pyramidal neurons also suggests a dysfunctional role for such elevated firing rates, which may shift the computational properties of this network (Wilson et al, 2006).

We tested whether treatments aimed at reducing excess neuronal activity in aged rats would improve memory performance. Boosting neuropeptide Y (NPY) 
neurotransmission, especially through Y2 receptors, has been shown to effectively attenuate neuronal excitability in seizure models (Foti et al, 2007; Morris et al, 2007), indicating a potential application to reduce elevated activity associated with neurocognitive aging. Hence, we first examined whether increasing NPY Y2 receptor activation via adeno-associated virus (AAV) delivered into the CA3 of the hippocampus would improve memory in cognitively impaired aged rats. We then tested two different pharmacological agents that are widely used antiepileptic agents, sodium valproate (VPA) and levetiracetam (LEV). In each case, improved behavioral performance was produced by these treatments in aged rats with identified cognitive impairment, suggesting that excess neural activity in the CA3 region contributes to behavioral memory deficits. A test of LEV in young adult rats showed no such benefit, further supporting efficacy in the condition of memory impairments in aging.

\section{MATERIALS AND METHODS}

\section{Subjects}

Aged, male Long-Evans rats were obtained at 8 to 9 months of age from Charles River Laboratories (Raleigh, NC) and housed in a vivarium at Johns Hopkins University until 24 to 26 months of age. Young rats obtained from the same source were housed in the same vivarium and tested at 6 months of age. All rats were individually housed at $25^{\circ} \mathrm{C}$ and maintained on a $12 \mathrm{~h}$ light/dark cycle. Food and water were provided ad libitum unless noted otherwise. The rats were examined for health and pathogen-free status throughout the experiments, as well as necropsies at the time of killing. All procedures in the current investigations were approved by the Institutional Animal Care and Use Committee in accordance with the National Institutes of Health directive.

\section{Background Behavioral Characterization}

All rats were screened in a standardized assessment of spatial cognition before the studies with experimental treatments. The background assessment used a wellestablished Morris water maze protocol as described in detail elsewhere (Gallagher et al, 1993). Briefly, the rats were trained for 8 days (three trials per day) to locate a camouflaged escape platform that remained at the same location throughout training in a water maze. Every sixth trial consisted of a probe trial (free swim with no escape platform) that served to assess the development of a spatially localized search for the escape platform. During these probe trials, a learning index was generated from the proximity of the rat to the escape platform and was used to define impairment in the aged rats. The learning index is the sum of weighted proximity scores obtained during probe trials, with low scores reflecting a search near the escape platform and high scores reflecting searches farther away from the platform (Gallagher et al, 1993). Cue training (visible escape platform) occurred on the last day of training to test for sensorimotor and motivational factors independent of spatial learning. Aged rats with impaired spatial memory performance (ie, those with learning index scores outside the young 'normative' range) but successful cued training performance were used for the studies as described below.

\section{Surgery and Treatments}

In the NPY experiment, memory-impaired aged rats were assigned to either NPY or control treatment $(n=6$ per group), with group assignment matched for learning index scores in the background characterization. The rats were injected with either an AAV vector that expresses and constitutively secretes NPY 13-36 (targeting Y2 receptors) or control substances (AAV-GFP or saline) bilaterally into the CA3 area of the hippocampus ( $2 \mu \mathrm{l}$ per side). That surgery, performed under isoflurane anesthesia, occurred 2 weeks before further behavioral assessment. Stereotaxic coordinates were $3.6 \mathrm{~mm}$ posterior to bregma, $3.0 \mathrm{~mm}$ lateral to midline, and $3.8 \mathrm{~mm}$ ventral to the skull surface. AAV-NPY 13-36 vector contained a chicken $\beta$-actin (CBA) promoter driving expression of a fragment of the NPY gene encoding amino acids 13-36 fused to the fibronectin signal sequence (FIB-NPY 13-36). The addition of the fibronectin signal sequence results in constitutive, unregulated secretion of NPY 13-36 protein from the transduced cells. Although the FIB-NPY 13-36 mRNA is readily detected, the secreted protein degrades rapidly making immunohistochemical detection difficult (Haberman et al, 2003). The AAV-GFP control vector contained the CBA promoter driving enhanced green fluorescent protein. AAV virus was made using standard procedures (Foti et al, 2007). All virus titers were approximately $1 \times 10^{12}$ particles per ml.

In an initial VPA experiment, memory-impaired aged rats were implanted subcutaneously in the intrascapular region with osmotic mini-pumps (ALZET, Durect, Cupertino, CA) with VPA (50 or $100 \mathrm{mg} / \mathrm{kg} /$ day; Sigma-Aldrich, St Louis, $\mathrm{MO}$ ) or saline vehicle starting 2 weeks before the intervention experiments. The VPA treatment at $100 \mathrm{mg} / \mathrm{kg} / \mathrm{day}$ via osmotic mini-pump was determined to produce a mean total VPA concentration of $10 \mu \mathrm{g} / \mathrm{ml}(\mathrm{SEM}=1.28 ; n=20)$ in the plasma of aged rats, a level that agrees with those reported by others when similar dosing with VPA was also chronically administered via osmotic mini-pumps (Stout et al, 2001). Other behavioral experiments used acute administration of VPA or LEV (Sigma-Aldrich or Tecoland, Edison, NJ) given by systemic injection 30-40 min before test sessions.

\section{Behavioral Assessment in the Water Maze}

Rats were trained and tested in a novel water maze environment to assess the effect of the treatments. The water maze used here was housed in a different building and was surrounded by curtains with a novel set of patterns relative to the maze used for initial assessment of cognitive status. The training protocol used in the NPY experiment and in an assessment of LEV consisted of six trials per day for 2 days to locate a submerged escape platform. On each trial, a rat was released in the maze from one of four equally spaced starting positions around the perimeter of the pool. The starting position varied from trial to trial. If the rat did not locate the escape platform within $60 \mathrm{~s}$ on any trial, the experimenter guided and placed the rat on the platform, where it remained for $20 \mathrm{~s}$. The rat was then removed from 
the platform and placed in a holding cage for another $40 \mathrm{~s}$ before the next trial. Approximately $24 \mathrm{~h}$ after the last training trial, a probe test in the absence of the escape platform was given to assess spatial memory.

The training protocol for the VPA experiment in the water maze was based on a modified water maze task that has been shown to be hippocampal-dependent (de $\mathrm{Hoz}$ et al, 2005; Steele and Morris, 1999). Unlike the traditional water maze protocol (such as that used in the background assessment and the NPY and LEV experiments) wherein the escape platform location remained constant throughout training, the escape platform location in this version of the task varied from day to day. During each day's training session, rats were given six trials to locate the submerged escape platform. At the end of the training session, the rats were returned to their home cages. After a 6-h delay, the rats were returned to the water maze for a retention trial with the submerged platform located in the same position as in the previous session of training trials. Each rat was given $60 \mathrm{~s}$ to locate the platform. Rats were pretrained on this protocol for 2 days, and then trained and tested in this manner for 3 consecutive days. Performance on these 3 days was averaged for analysis using the escape latency and path to locate the platform as measures of retention; spatial bias in a free swim was not used because the repeated training protocol encourages rats to search for a new location when escape is not readily achieved.

\section{Behavioral Assessment in the Radial Arm Maze}

A radial arm maze task was used to assess effects of acute drug treatment with LEV, VPA, and the combination of those drugs. This protocol allowed within-subject assessment across drugs at different doses and in combinations. The radial maze consisted of eight arms projecting from each side of an octagonal center platform, with food well located at the distal end of each arm. Plexiglas blocks could be positioned to prevent entry into any arm. Extra-maze cues were provided in the room surrounding the maze and illumination was provided by an overhead light.

Pre-training, as described in detail in Chappell et al (1998), consisted of habituation, standard win-shift training, and win-shift training with delays interposed between information and memory test phases. Drug treatments began 2 days after the completion of pre-training. Three arms were blocked at the beginning of each trial (information phase). The identity and configuration of the blocked arms varied across trials. Food-deprived rats were allowed to retrieve food reward (Kellogg's Froot Loops cereal) from the five unblocked arms. The rat was then removed from the maze for $60 \mathrm{~min}$ (retention interval), during which time the barriers on the blocked arms were removed allowing access to all eight arms. Rats were then placed back onto the center platform and allowed to retrieve the remaining food rewards (memory test phase). An error consisted of returning to an arm (all four paws on the arm) from which food had already been obtained. Memory-impaired aged rats $(n=10)$ were first tested with a series of LEV doses in ascending/descending order; each dose was thus tested twice, with one washout day in between each determination. At the completion of LEV assessment, the same rats $(n=9$, because one rat died) were tested with a series of VPA doses in the same fashion as that for LEV assessment with performance at each dose tested twice. About a week after the completion of the individual drug assessments, the rats were given combination drug dosing in which ineffective doses of LEV and VPA in monotherapy were administered in combination $(n=8$, one rat had to be dropped because of health deterioration). The number of errors made in the retention phase after the 1 -h delay was used to assess memory performance.

To determine whether treatment efficacy is age specific, young rats $(n=8)$ were tested with varying doses of LEV in the same fashion as that in the aged rats, except that a 5-h retention interval was used to approximate the number of errors committed by memory-impaired aged rats under drug-free condition as described above.

\section{In Situ Hybridization}

At the end of the NPY experiment, the brains of the rats were harvested following perfusion. A riboprobe template targeting the FIB NPY 13-36 sequence was generated by PCR from rodent whole hippocampus RNA and modified to contain SP6 and T7 RNA polymerase sites. ${ }^{35}$ S-UTP-labeled riboprobe was generated using the Maxiscript kit (Ambion). Brain sections $(40 \mu \mathrm{m})$ from across the entire extent of the hippocampus were hybridized overnight at $60^{\circ} \mathrm{C}$ in buffer containing the radiolabeled riboprobe. Hybridized sections were then extensively washed and mounted onto slides.

\section{RESULTS}

\section{Background Assessment of Cognitive Status}

Rats were tested for cognitive status before the intervention experiments using a protocol developed in this study population. Figure 1 shows the mean learning index of memory-impaired aged rats $(n=67)$ subsequently used in the current experiments, and for comparison, the learning index of young rats $(n=122)$ that were tested concurrently with these aged rats during background characterization. Higher learning index scores signify worse performance by reflecting search at a greater distance from the escape

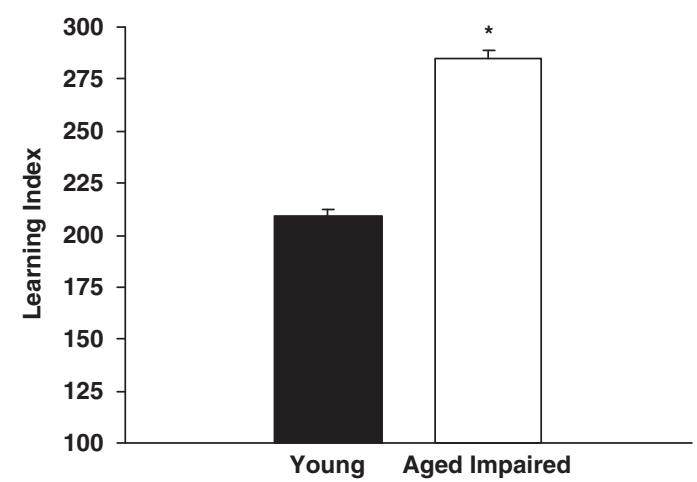

Figure I Background behavioral characterization. The cognitive status of aged and young rats was assessed in a standardized water maze task before intervention experiments. Higher learning index scores signify worse performance by reflecting search at a greater distance from the escape location during memory probe tests. Aged rats that performed beyond the normative range of young adult rats in this study population were selected for the present studies. $* p=0.001$. 
location during memory probe tests. As only aged rats that performed beyond the normative range of young adult rats in this study population were selected for the subsequent experiments, it is not surprising that the performance of these aged rats was significantly impaired relative to the young rats, $t(187)=13.97, p=0.001$.

\section{Spatial Memory Under AAV-NPY Treatment}

Rats were assigned to treatment groups based on the background characterization (mean learning indexes were $273(\mathrm{SEM}=14.5)$ and $279(\mathrm{SEM}=14.7)$ for experimental and control groups, respectively). Two weeks after NPY or control injection, aged rats were trained and tested in a novel water maze environment. Figure 2 a shows the average escape latencies over four blocks of training trials (three trials per block). An analysis of variance (ANOVA) with repeated measures showed significant improvement over the course of training as indicated by decreased escape latencies in a main effect of Trial Block, $\mathrm{F}(3,30)=3.24$, $p=0.036$. Rats in the NPY group also showed significantly better performance than the age/impairment-matched control group as indicated by a significant group effect, $\mathrm{F}(1,10)=6.28, p=0.031$. Even though the group $\times$ trial block interaction was not significant, $F(3,30)=1.12$, $p=0.358$, the overall better performance of the NPY group was not evident on the first training trial, $t(10)=0.537$, $p=0.603$. The group difference in escape latencies was also not because of swim speed performance because there was no significant difference on that measure during training trials for rats in the two groups $(\mathrm{NPY}=17.1 \mathrm{~cm} / \mathrm{s}$, $\mathrm{SEM}=1.37$; control $=15.6 \mathrm{~cm} / \mathrm{s}, \mathrm{SEM}=2.48$ ).

A probe test was subsequently given $24 \mathrm{~h}$ after training in which the escape platform was removed. As expected for aged rats characterized with impairment, the control group showed no evidence of memory, searching a similar amount of time in all quadrants of the maze (Figure 2b). In contrast, the NPY group showed retention for the escape platform location as indicated by a strong spatial bias for the target quadrant. An ANOVA with repeated measures showed a marginally significant group $\times$ quadrant interaction overall, $\mathrm{F}(3,30)=2.91, p=0.051$. Further analysis confirmed that rats in the NPY group showed a spatial bias for the

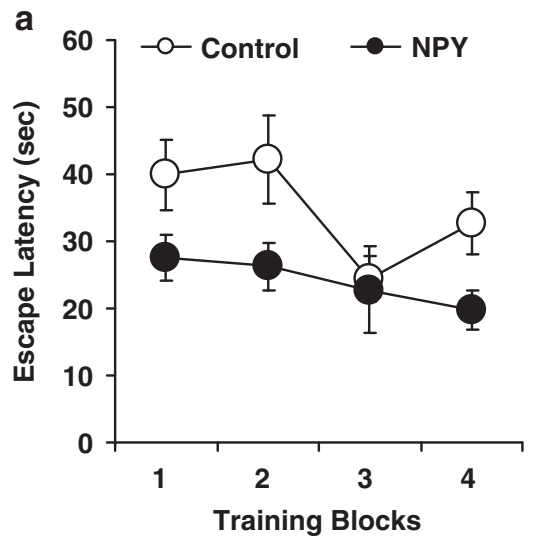

target quadrant, $t(5)=2.86, p=0.035$ (compared with the average search in the other quadrants). In contrast, that analysis showed no significant spatial bias in the performance of rats in the control group, $t<1$. Finally, rats in the NPY group spent significantly more time in the target quadrant compared with those in the control group, $t(10)=2.33, p=0.042$.

Photomicrographs of hippocampal sections of aged rats in the NPY group confirmed that FIB-NPY 13-36 mRNA was present in the area of AAV injection (Figure 3), whereas control brains showed absence of specific probe hybridization.

\section{VPA Treatment on Memory Performance in the Water Maze Task}

The effect of chronic treatment with the antiepileptic drug, VPA, was assessed in separate groups of aged rats implanted with osmotic mini-pumps as described in Materials and methods section $(50 \mathrm{mg} / \mathrm{kg}, n=9 ; 100 \mathrm{mg} /$ $\mathrm{kg}, n=13$; vehicle treatment, $n=17)$. Group assignment was matched for learning index scores (291 (SEM = 10.8), $290(\mathrm{SEM}=5.4)$, and $297(\mathrm{SEM}=7.7)$ for $\mathrm{VPA} 50 \mathrm{mg} / \mathrm{kg}$, VPA $100 \mathrm{mg} / \mathrm{kg}$, and vehicle groups, respectively). In this study, rats were trained to new spatial locations in each day's training session and a retention test occurred at a $6 \mathrm{~h}$ delay. Figure 4 shows the path length (a) and escape latency (b) data for rats that received treatment of vehicle (VEH), VPA at $50 \mathrm{mg} / \mathrm{kg} /$ day (VPA 50 ), or VPA at $100 \mathrm{mg} / \mathrm{kg} /$ day (VPA 100) averaged over three new escape locations. During training, rats in all three groups improved within the day's session, attaining similar proficiency in performance by the last training trial. For path length, an ANOVA with repeated measures showed a significant main effect of trial, $\mathrm{F}(5,180)=19.94, p=0.001$. There was also a main effect of group, $\mathrm{F}(2,36)=4.02, p=0.027$, reflecting somewhat longer paths in rats that received VPA at $50 \mathrm{mg} / \mathrm{kg}$ relative to the groups that received vehicle or VPA at $100 \mathrm{mg} / \mathrm{kg}$. The three groups however were not different from each other at the end of training, $p>0.304$ (Trial 6). Similar results were obtained in the analysis of escape latency. Rats took progressively less time to escape over trials, $\mathrm{F}(5,180)=6.27, p=0.001$, with a trend for group

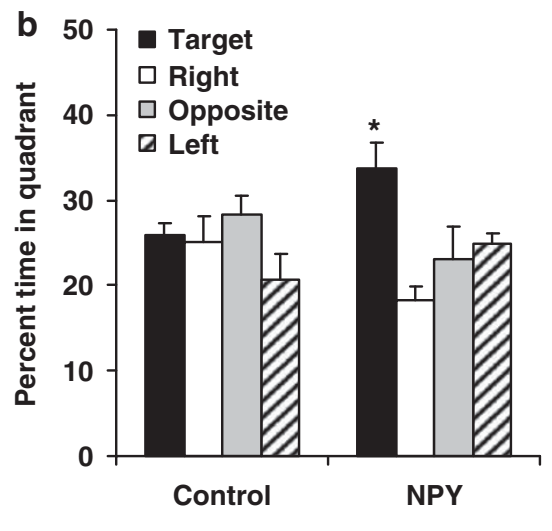

Figure 2 Behavioral assessment of NPY treatment. Memory-impaired aged rats that received AAV-NPY I3-36 injections into the CA3 of the hippocampus 2 weeks before, performed better than control rats during water maze training (a), and showed stronger memory retention for the escape platform location in a probe trial $24 \mathrm{~h}$ after training (b). See text for details of data analysis. 
Control

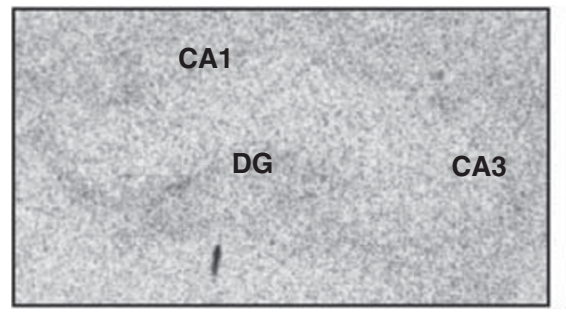

FIB-NPY

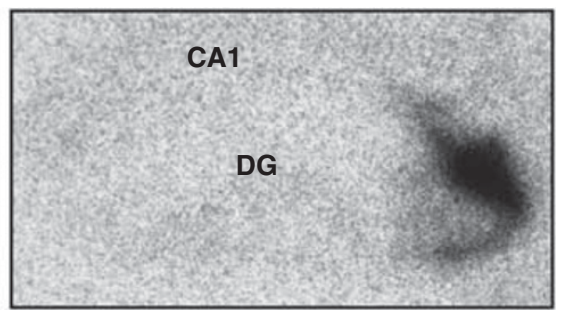

Figure 3 AAV-NPY transduction in the hippocampus. Photomicrographs of coronal hippocampal sections of aged rats showing FIB-NPY I3-36 mRNA (right panel) was present in the NPY group, and the absence of specific probe hybridization in the brain of a control rat (left panel). In testing for the FlBNPY in situ probe, we also generated and hybridized a sense control probe. This control probe showed very little signal as expected (not shown). DG, dentate gyrus.
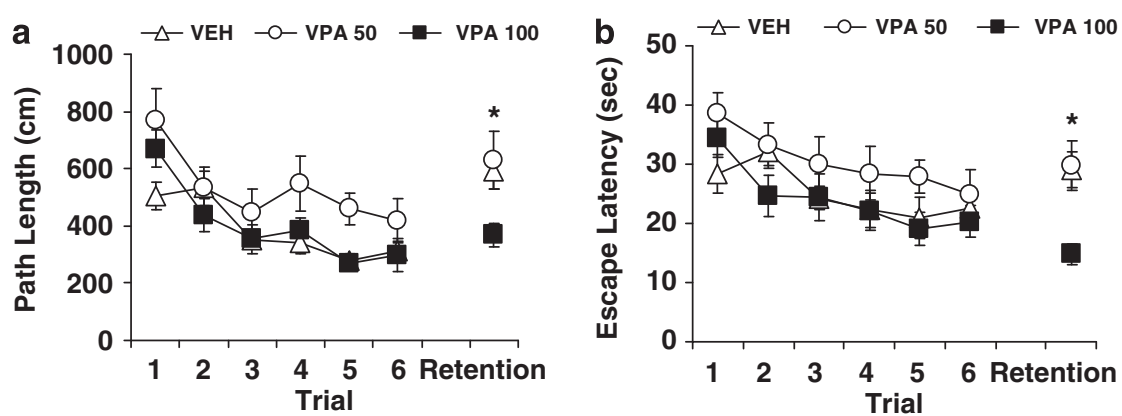

Figure 4 VPA assessment in the water maze. Memory-impaired aged rats that received chronic treatment with VPA or saline performed at a comparable level at the end of the water maze training phase (Trial 6), but those treated with VPA at $100 \mathrm{mg} / \mathrm{kg} /$ day (VPA I00) showed less forgetting as measured by path length (a) and escape latency (b) after a 6-h delay (Retention Trial) compared with their counterparts treated with saline (VEH) or VPA at 50 mg/kg/day (VPA 50). See text for details of data analysis.

differences, $\mathrm{F}(2,36)=2.81, p=0.073$, but no performance difference among the groups at the end of training sessions, $p>0.661$.

The rats were given a retention trial after a 6-h delay to assess memory of the previous session's learning. Better retention was evident in rats treated with VPA at $100 \mathrm{mg} / \mathrm{kg} /$ day for both escape latency and path. A one-way ANOVA indicated a significant group effect, $\mathrm{F}(2,36)=4.258$, $p=0.022$ for path length, and $\mathrm{F}(2,36)=7.606, p=0.002$ for escape latency. Further group comparisons confirmed that rats in the VPA 100 group had significantly shorter path lengths and escape latencies than those in the VEH and VPA 50 groups (all $p$-values $<0.05$ ). The VEH and VPA 50 groups were not different from each other on either measure, $p$-values $>0.927$. Taken together, these results indicate that chronic VPA treatment dose-dependently improves retention of new spatial information in aged rats previously characterized with hippocampal-dependent cognitive impairment.

\section{VPA, LEV, and Combination Therapy on the Radial Arm Maze Task}

To further assess the effects of antiepileptic drugs in aged impaired rats, we used a radial arm maze task that allowed a within-subject design to compare two different compounds, VPA and LEV. This task also extended the behavioral assessment to spatial memory using appetitive motivation. An independent set of aged rats $(n=10)$, which were characterized with impairment in the background water maze assessment (mean learning index $=266, \mathrm{SEM}=7.1$ )

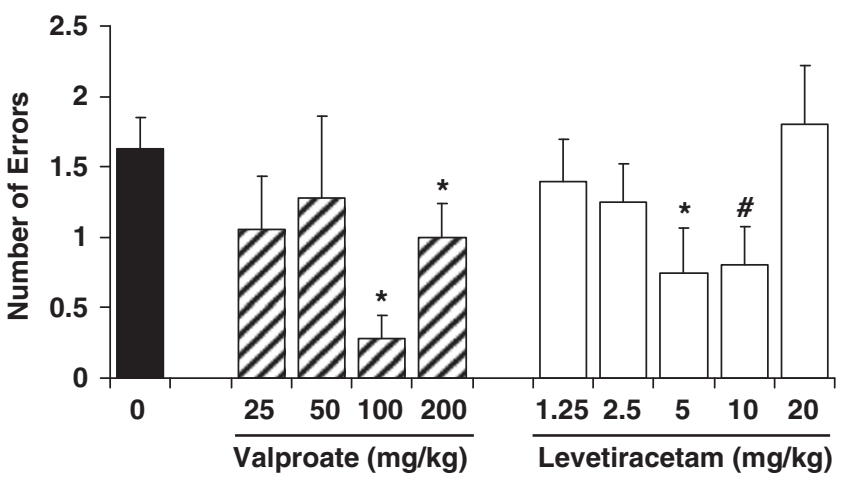

Figure 5 VPA and LEV assessment in the radial maze. Efficacious doses of VPA and LEV to improve memory performance of aged rats with cognitive deficit were determined using a within-subject design in an eightarm radial maze. Over the course of the study, the same rats were tested repeatedly with vehicle saline $(0 \mathrm{mg} / \mathrm{kg})$, VPA, and LEV. Post-delay memory errors were significantly lower than vehicle baseline when rats were treated with VPA at 100 and $200 \mathrm{mg} / \mathrm{kg}$, as well as with LEV at 5 and $10 \mathrm{mg} / \mathrm{kg}$. ${ }^{*} p<0.050$ and ${ }^{\#} p=0.05$ I compared to vehicle treatment $(0 \mathrm{mg} / \mathrm{kg})$.

served as subjects. Figure 5 shows the memory performance of those rats treated with varying doses of VPA by subcutaneous (s.c.) injection and LEV by intraperitoneal (i.p.) injection as indexed by the number of errors made during post-delay memory tests. Differences in memory performance were evident under VPA treatment as a function of dose, $\mathrm{F}(4,32)=2.87, p=0.039$. The VPA doses at 100 and $200 \mathrm{mg} / \mathrm{kg}$ significantly reduced errors relative to vehicle, $t(8)=6.54, p=0.001$ and $t(8)=2.39, p=0.044$, 
respectively. LEV also appeared to decrease errors in the post-delay tests. An overall analysis of LEV administration in the aged rats revealed differences in performance as a function of dose, $\mathrm{F}(5,45)=2.38, p=0.053$, with rats making fewer errors on 5 and $10 \mathrm{mg} / \mathrm{kg}$ of LEV compared with saline, $t(9)=3.53, p=0.006$ and $t(9)=2.25, p=0.051$, respectively. These results therefore confirm the initial findings in the water maze with VPA, extend the improvement seen with that treatment to an appetitive spatial memory task, and suggest that another antiepileptic drug, LEV, has similar benefit in a low-dose range.

We also tested combinations of LEV and VPA at subthreshold doses (when administered individually) to assess the possibility that these drugs might improve memory when given in combination. Before those drug tests, we first re-established baseline performance with a combination of i.p and s.c. saline injections. Figure 6 a shows the results of combination doses and the comparison vehicle treatment. Compared with vehicle, the combination of VPA $50 \mathrm{mg} / \mathrm{kg}$ and LEV $2.5 \mathrm{mg} / \mathrm{kg}$, as well as the combination of VPA $50 \mathrm{mg} / \mathrm{kg}$ and LEV $1.25 \mathrm{mg} / \mathrm{kg}$, were effective at significantly reducing memory errors, $t(7)=2.49, \quad p=0.042$, and $t(7)=2.68, p=0.032$, respectively. The combination of VPA $25 \mathrm{mg} / \mathrm{kg}$ and LEV $2.5 \mathrm{mg} / \mathrm{kg}$, however, did not differ from vehicle. We plotted those data in an isobologram. As indicated in the isobologram (Figure 6b), the dose combination of VPA $50 \mathrm{mg} / \mathrm{kg}$ and LEV $1.25 \mathrm{mg} / \mathrm{kg}$ falls below the line/isobole of additivity, suggesting superadditive or synergistic interaction between VPA and LEV in that combination of doses.

\section{LEV Treatment on Memory Performance in the Water Maze Task}

In a final experiment, acute treatment with $\mathrm{LEV}$ at $10 \mathrm{mg} / \mathrm{kg}$ was given to impaired aged rats $(n=6$; mean learning index $=280, \mathrm{SEM}=6.6$ ) during water maze training using a within-subject design to compare drug performance with saline. Hence upon completion of assessing the first treatment condition (drug or vehicle, counterbalanced), the rats received the other treatment starting the next day in the same maze but surrounded by a new set of curtains with a novel configuration/color of cues, and a new escape platform location. Figure $7 \mathrm{a}$ shows performance during training trials. An ANOVA with repeated measures revealed that the rats improved during training as their escape latencies significantly decreased over trials, $\mathrm{F}(3,15)=5.58$, $p=0.009$. Although performance appears better under drug than saline at the end of training, no statistical difference was revealed involving treatment; that is, no significant treatment $\times$ trial block interaction $(p>0.25)$ or main treatment effect $(p>0.60)$.

A memory probe test was given $24 \mathrm{~h}$ after the end of training (data in Figure 7b). An ANOVA with repeated measures showed a strong trend for a treatment $\times$ quadrant interaction, $\mathrm{F}(3,15)=3.14, p=0.057$, in which LEV treatment during training augmented subsequent retention for the escape platform location. A spatially localized search was evident in time spent in the target quadrant, which was significantly greater when rats had been treated with LEV compared with saline, $t(5)=2.80$, $p=0.038$. Overall then, these results indicate that LEV treatment at $10 \mathrm{mg} / \mathrm{kg}$ benefits long-term spatial memory in aged rats that are otherwise impaired.

\section{LEV Treatment on Memory Performance in Young Rats}

On the basis of our hypothesis of targeting excess hippocampal activity, we expected that these treatments might only benefit aged rats but not young rats. In a study run in parallel with the aged rats, injection of AAV-NPY into the CA3 of young rats (6 months old) produced no improvement in the water maze performance beyond that of control rats (data not shown). That study however was limited by strong memory performance in the young control rats, thus possibly precluding the detection of improvement in the experimental treatment group. To address that limitation, we ran a separate study with a new batch of young rats using the radial arm maze and imposing a lengthy delay between information and test phase (5-h delay) to attain a number of errors similar to those of aged rats at the shorter delay. Before treatment, these young rats had received the same background water maze assessment (mean learning index $=191, \mathrm{SEM}=10.1$ ) as the aged rats.
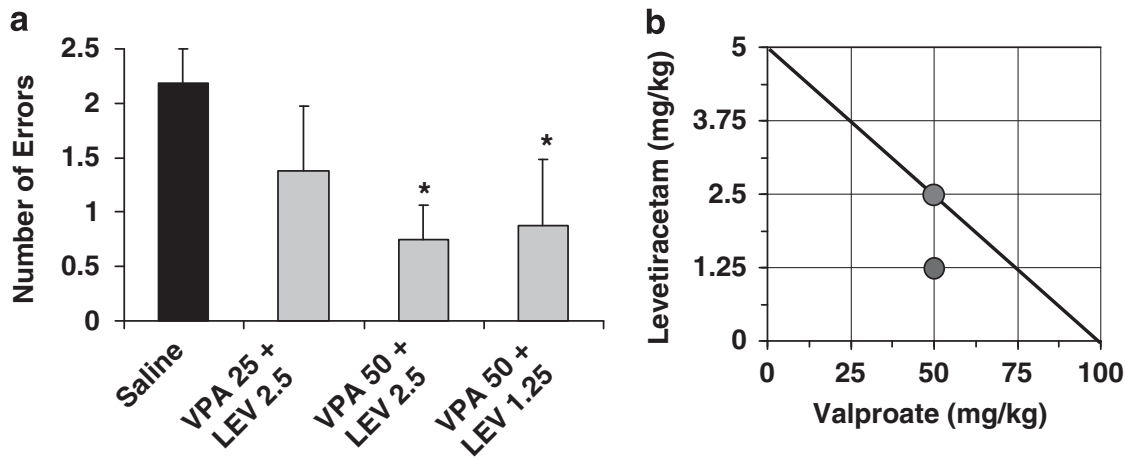

Figure 6 Combination VPA/LEV assessment in the radial maze. Combination therapy consisting of VPA and LEV was assessed in the radial arm maze after each drug was tested individually over a range of doses. (a) Baseline performance was established with combination saline injections, and sub-threshold doses of each drug (VPA at 25 or $50 \mathrm{mg} / \mathrm{kg}$, and LEV at 1.25 or $2.5 \mathrm{mg} / \mathrm{kg}$ ) were administered in combination. To facilitate interpretation, effective combinations were then plotted on an isobologram (b). The diagonal straight line is the line of additivity, anchored on each axis by the lowest effective doses of VPA and LEV when assessed individually. Efficacious combinations at lower sub-threshold doses that fall on the line are interpreted accordingly as a simple additive effect, as is the case for the treatment of VPA $50 \mathrm{mg} / \mathrm{kg}$ combined with LEV $2.5 \mathrm{mg} / \mathrm{kg}$. The dose combination of VPA $50 \mathrm{mg} / \mathrm{kg}$ and LEV I.25 mg/kg falls below the line of additivity, suggesting super-additive or synergistic interaction between the two drugs. 

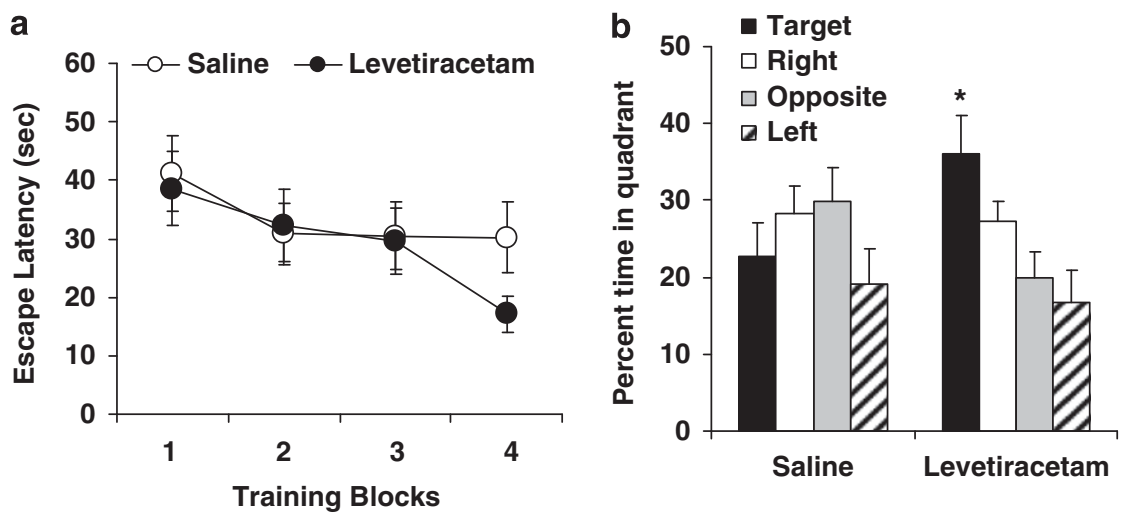

Figure 7 LEV assessment in the water maze. Memory-impaired aged rats were injected with LEV at $10 \mathrm{mg} / \mathrm{kg}$ or saline vehicle before training in the water maze (a). Better memory retention was observed under LEV than saline treatment $24 \mathrm{~h}$ later in a probe test (b). $* p<0.050$ compared with percent time spent in target quadrant under saline treatment.

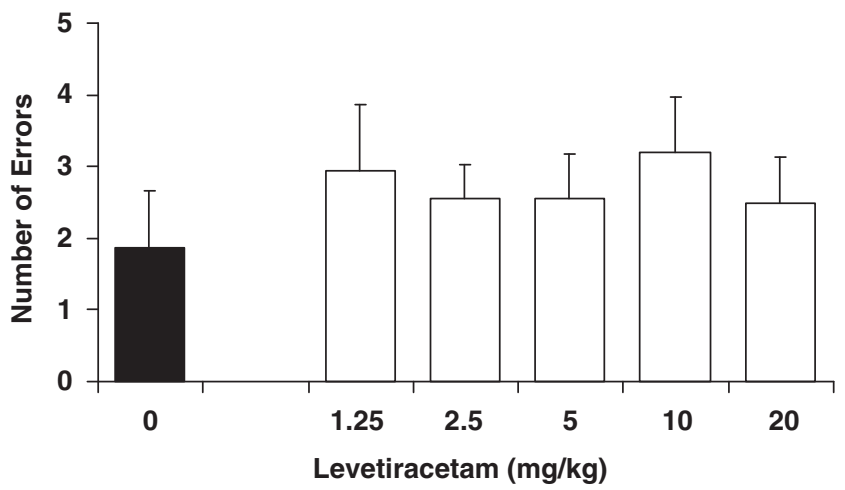

Figure 8 LEV treatment in young rats. Young rats were treated with LEV at varying doses before testing in the radial arm maze using a 5-h retention delay. No differences in memory performance were found as a function of dose.

They were then tested on the radial arm maze with a series of LEV doses in the same fashion as that in the aged rat study. Figure 8 shows the memory performance of young rats treated with varying doses of LEV as indexed by the number of errors made during post-delay memory tests. No significant differences in performance were found as a function of dose, $F(5,35)=0.46, p=0.806$. These data indicate that this pharmacological treatment aimed at reducing excess hippocampal activity only benefits memory performance in aging.

\section{DISCUSSION}

The studies reported here were designed to test the hypothesis that excess neural activity in the hippocampus of aged rats contributes to memory impairment. We first overexpressed an inhibitory neuropeptide (NPY 13-36) targeting the CA3 region of the hippocampus of aged rats using methods shown to attenuate aberrant excitability in limbic seizure models. We also tested two widely used antiepileptic agents administered systemically. In each case, aged rats with established memory impairment showed improved behavioral performance in spatial memory tasks. The converging evidence for benefits of these treatments indicates that excess excitation in the hippocampus may have a key role underlying age-related impairment in hippocampal-dependent memory processes as proposed for this model (Wilson et al, 2006).

Aged rats in the current research came from a study population with well-characterized individual differences in memory impairment. Importantly, previous studies have shown a high degree of test-retest reliability for the presence and severity of those individual differences in behavioral assessments; aged rats that fall outside the range of normative young performance in the standardized assessment show a similar behavioral profile in subsequent tests of hippocampal-dependent memory (Colombo et al, 1997; Robitsek et al, 2008). Such evidence increases confidence that the assignment of subjects based on the standardized background assessment ensured a valid test of treatment efficacy in experiments using between group comparisons. In addition, the data obtained in the experiments using within-subject designs support the reliability of individual differences because impairment was routinely evident under vehicle treatment in those studies, as discussed further below.

The hypothesis examined here is also grounded in much background research in this model showing that behavioral status in the aged rat study population is strongly correlated with neurobiology in the underlying circuitry of the hippocampal formation. Although aged rats in this study population maintain similar number of neurons and largely preserved synaptic connections in the medial temporal lobe system (Geinisman et al, 2004; Rapp and Gallagher, 1996; Rapp et al, 2002; Smith et al, 2000), functional alterations are closely tied to age-related cognitive decline. The striking increase in neural activity in the CA3 region led to a proposal for the basis of cognitive impairment, which links that excess activity to loss of encoding that normally depends on the CA3/dentate gyrus subdivisions of the hippocampal network (Wilson et al, 2006). As the majority of synaptic input to the CA3 pyramidal neurons comes from the excitatory recurrent CA3 collaterals, elevated activity would be expected to promote pattern completion, a computational function attributed to the CA3 autoassociative network that favors retrieval of previously stored representations (Guzowski et al, 2004; Treves and Rolls, 1994). In addition, the joint occurrence of elevated activity and reduced plasticity is well established in studies of neural 
networks, including the hippocampus; synaptic plasticity most readily occurs in an optimal range of activity, and thresholds for synaptic modification can rise in conditions of excess activity (see Abraham et al, 2001 for example, and Abraham, 2008 for recent review). Recording data from hippocampal neurons in this study population are in agreement with this concept linking excess activity to a failure to encode new information in the CA3 network (Wilson et al, 2005). By that view, experimental treatments that lower activity would be expected to lower the threshold for synaptic modification to allow the encoding of new information.

As an initial test of this idea, we locally expressed an inhibitory neuropeptide, NPY13-36, which has been shown elsewhere to be effective in modulating neuronal excitability under both normal and epileptic conditions (Foti et al, 2007; McQuiston and Colmers, 1996; Vezzani et al, 1999). NPY13-36 preferentially activates the Y2 receptor subtype which has a predominant role in mediating the NPYinduced inhibition of glutamate release in the CA1 and CA3 of the hippocampus (El Bahh et al, 2005; Silva et al, 2005). We engaged the inhibitory property of $\mathrm{Y} 2$ receptor activation by constitutively expressing NPY 13-36 via an $\mathrm{AAV}$ vector delivered into the CA3; this resulted in significantly improved hippocampal-dependent memory in the aged rats. In situ histochemistry for the vector-produced mRNA confirmed that expression of NPY13-36 was restricted to the hippocampus and centered primarily on the CA3, likely providing only local control of inhibition in that area. Because no elevated activity is observed in the CA1 subregion of the hippocampus in our aging model (Wilson et al, 2005), and CA3 connections (Shaffer collaterals) onto CA1 neurons have reduced efficacy in the aged brain (Lister and Barnes, 2009), boosting inhibition in the adjacent CA1 area would not be expected to have beneficial effects on network function. These data therefore support the view that controlling excess neural activity in the CA3 of aged rats improves hippocampal network function and hippocampal-dependent memory performance, and serves as a proof of principal for further exploration into more clinically accessible methods of modulating excess neural activity.

Subsequent tests centered on the same rationale of targeting excess excitation by administering two widely used antiepileptic agents, VPA and LEV, which each showed the ability to improve spatial memory in aged rats. In the first of those studies, VPA was assessed in a spatial memory task when aged memory-impaired rats learned new escape platform locations in each day's session in the water maze. Rats implanted with osmotic mini-pumps to administer $100 \mathrm{mg} / \mathrm{kg}$ had substantial savings after a $6 \mathrm{~h}$ delay, escaping with similar proficiency relative to the end of the previous session's training trials. Those rats differed from the control vehicle group and a group receiving a lower dose of VPA, which showed little savings for previous training after the delay. In a subsequent study using a within-subject design, VPA doses of 100 and $200 \mathrm{mg} / \mathrm{kg}$ significantly improved memory performance on the radial maze after a 1-h delay. The improvement obtained with effective doses of VPA reduced errors to levels typically seen in young rats tested on this task (Chappell et al, 1998). Together those experiments show a benefit from VPA treatment across spatial memory tasks with different motivational and performance demands. It is important to note that blood levels of VPA in rats at the doses effective for neurocognitive aging $(10 \mu \mathrm{g} / \mathrm{ml}$; see Materials and methods section for details) are well below those needed for antiepileptic efficacy to control seizure activity $(225 \mu \mathrm{g} / \mathrm{ml}$ and above; Tulloch et al, 1982). Indeed, high dosing might be less effective for cognitive improvement in aging by producing more pronounced and widespread effects on brain function.

Similar to VPA, LEV was also found to improve memory performance on the radial maze at doses substantially lower than those used in seizure models. Significant improvement was observed at 5 and $10 \mathrm{mg} / \mathrm{kg}$, but not at a higher dose of $20 \mathrm{mg} / \mathrm{kg}$. Typical antiepileptic doses of LEV in rodent seizure models are in a range of $50-150 \mathrm{mg} / \mathrm{kg}$ (eg, Ji-qun et al, 2005; Stratton et al, 2003). In addition, the two antiepileptic compounds VPA and LEV exhibited benefit in combination at doses that were otherwise ineffective. Comparing the dose response for the individual drugs with the combinations provided evidence for drug synergy rather than merely additive effects. As an extension of the data obtained in the radial maze, improved retention over a $24 \mathrm{~h}$ delay was confirmed with LEV at $10 \mathrm{mg} / \mathrm{kg}$ in an independent set of memory-impaired aged rats trained in a novel water maze environment. Finally, as expected under the view that benefit is because of dampening excess activity, we found no improvement in memory performance when young adult rats were given LEV.

Although the route by which VPA and LEV exert antiepileptic efficacy is not known with certainty, and those mechanisms may not directly apply to the doses used in the current studies, these two compounds share little apparent mechanistic overlap. The efficacy of VPA as an antiepileptic potentially involves a variety of mechanisms, including increased $\gamma$-amino butyric acid (GABA)-ergic transmission, increased uptake of excitatory amino acids, and blockade of voltage-gated sodium channels (Perucca, 2002). At the low doses found to be effective in the current research, the most likely mechanisms involve control of excitatory transmission by reducing extracellular accumulation of glutamate and increased GABAergic transmission. An upregulation of hippocampal glutamate transport occurs in rats treated with VPA (Hassel et al, 2001). We confirmed this finding at doses used in our aged rats and further observed that low doses of VPA administered systemically increase the hippocampal expression of GLT1b, an isoform of the glutamate transporter ( $M$ Gallagher, unpublished data). In contrast to the localization of GLT1a in astrocytes, GLT1b is localized to neurons in association with the post-synaptic density (González-González et al, 2008). Thus, VPA may permit the regulation of excitatory transmission at synapses that drive neuron activity in addition to boosting inhibition. LEV is thought to mediate its effects via a synaptic vesicle protein Sv2a, which renders primed synaptic vesicles competent for activity-dependent (calcium-mediated) release (Chang and Südhof, 2009). At concentrations of up to $10 \mu \mathrm{M}$, LEV does not show binding affinity for a variety of known receptors, such as those associated with benzodiazepines, GABA, glycine, and NMDA ( $N$-methyl$\mathrm{D}$-aspartate). In vitro and in vivo recordings of epileptiform activity from the hippocampus have shown that LEV inhibits burst firing. In vivo data also show that LEV opposes a pilocarpine-induced increase of the 
orthodromically activated population spike in the CA3 region of hippocampus (Klitgaard et al, 2003). Synergistic activity has also been noted for use of VPA and LEV in combination in studies of seizure models (Kaminski et al, 2009).

Current data suggest that modifying excitatory-inhibitory balance through different routes can ameliorate behavioral memory impairment in aging. The benefit observed with these different treatments suggests that neural activity and encoding properties in the hippocampal network are ameliorated by targeting excess activity. Studies to directly monitor such effects, using electrophysiological recordings in awake behaving rats, are beyond the scope of the current investigation, but will be informative as to whether such restoration is a final common pathway for behaviorally effective treatments in this model.

The functional significance of aberrant activity in the hippocampus may not be limited to animal models of aging but may also have direct implications for memory decline experienced during aging in humans. A number of investigations using functional neuroimaging in human aging have reported increased activation in the medial temporal lobe during memory encoding. This finding has now been observed in patients with mild cognitive impairment (MCI; Celone et al, 2006; Dickerson et al, 2004; Dickerson et al, 2005; Hämäläinen et al, 2007), in older individuals with genetic or familial risk for developing Alzheimer's disease (Bassett et al, 2006; Bookheimer et al, 2000), and in aged subjects showing poor performance in memory but not sufficiently impaired to meet a diagnosis of MCI (Miller et al, 2008a). The original view of such increased activation was that it served a beneficial function by recruiting additional resources to provide 'compensation' for a failing neural network. A possible detrimental role of excess activation in humans, however, has been suggested by findings in follow up with MCI patients (Dickerson et al, 2004; Miller et al, 2008b). Specifically, Miller and colleagues (2008b) found that higher activation in the hippocampus, but not in signals imaged elsewhere in the brain, predicted greater subsequent cognitive decline $4-8$ years after initial evaluation. Consequently, those authors suggested that elevated hippocampal activation might serve as a therapeutic target for early intervention in cognitive decline. An assessment of whether reduction in elevated hippocampal activation in humans leads to worse memory performance, as suggested by a compensation view, or improves performance, consistent with the animal data obtained here, would provide a needed experimental test of the alternative interpretations now existing in the clinical literature. If the condition in humans' parallels the excess activity observed in studies of aged animals with memory impairment, such a test would be feasible using FDA approved compounds (VPA and LEV) that are effective in aged animals, albeit at lower doses than those used for other clinical indications.

Not much more than a decade ago it was widely believed that loss of neurons in the brain was the cause of memory impairment associated with aging. Now it is generally accepted that memory decline is predominantly a functional disorder in aged brains that are largely structurally intact. This view gives hope to the prospect for corrective therapies that will aid in the restoration of function in the aged brain. Many recent approaches to this problem have focused on boosting mechanisms underlying neural plasticity in young adults (Josselyn and Nguyen, 2005; Rose et al, 2005 for reviews). The current research supports a novel approach based on a disorder specific to the condition of neurocogntive impairment in the aged brain. As such, the findings may point to novel targets for therapies in this indication.

\section{ACKNOWLEDGEMENTS}

This research was supported by National Institute of Neurological Disorders and Stroke Grant NS-35633 to Thomas J McCown and National Institute of Aging Grant P01-AG-09973 to Michela Gallagher.

\section{DISCLOSURE}

No pharmaceutical company or private entity provided support for the present research. The products and services described in this publication are licensed to and are being developed by AgeneBio. Dr Michela Gallagher is the founder and Chairman of AgeneBio. Dr Gallagher owns company stock, which is subject to certain restrictions under University policy. The terms of this arrangement are being managed by the Johns Hopkins University in accordance with its conflict of interest policies. The authors (Koh, Haberman and Gallagher) are inventors on Johns Hopkins University intellectual property with patents pending that is under option to license to AgeneBio. Dr Gallagher serves as a member of the Board of Scientific Counselors to the National Institute on Aging and is also a member of the Scientific Advisory Board of the Stanley Center at the Broad Institute. Otherwise, she has had no consulting relationships with other public or private entities in the past 3 years and has no other financial holdings that could be perceived as constituting a potential conflict of interest. Drs. Ming Teng Koh, Rebecca Haberman, Stacey Foti, and Thomas J McCown have received, except for income received from their primary employer, no financial support or compensation from any individual or corporate entity over the past 3 years for research or professional services, and have no financial holdings that could be perceived as constituting a potential conflict of interest.

\section{REFERENCES}

Abraham WC (2008). Metaplasticity: tuning synapses and networks for plasticity. Nat Rev Neurosci 9: 387-399.

Abraham WC, Mason-Parker SE, Bear MF, Webb S, Tate WP (2001). Heterosynaptic metaplasticity in the hippocampus in vivo: a BCM-like modifiable threshold for LTP. Proc Natl Acad Sci USA 98: 10924-10929.

Bassett SS, Yousem DM, Cristinzio C, Kusevic I, Yassa MA, Caffo BS et al (2006). Familial risk for Alzheimer's disease alters fMRI activation patterns. Brain 129: 1229-1239.

Bookheimer SY, Strojwas MH, Cohen MS, Saunders AM, PericakVance MA, Mazziotta JC et al (2000). Patterns of brain activation in people at risk for Alzheimer's disease. $N$ Engl J Med 343: 450-456.

Celone KA, Calhoun VD, Dickerson BC, Atri A, Chua EF, Miller SL et al (2006). Alterations in memory networks in mild cognitive impairment and Alzheimer's disease: an independent component analysis. J Neurosci 26: 10222-10231.

Chang WP, Südhof TC (2009). SV2 renders primed synaptic vesicles competent for Ca2+ -induced exocytosis. J Neurosci 29: 883-897. 
Chappell J, McMahan R, Chiba A, Gallagher M (1998). A re-examination of the role of basal forebrain cholinergic neurons in spatial working memory. Neuropharmacology 37: 481-487.

Colombo PJ, Wetsel WC, Gallagher M (1997). Spatial memory is related to hippocampal subcellular concentrations of calciumdependent protein kinase $\mathrm{C}$ isoform in young and aged rats. Proc Natl Acad Sci USA 94: 14195-14199.

de Hoz L, Moser EI, Morris RG (2005). Spatial learning with unilateral and bilateral hippocampal networks. Eur J Neurosci 22: 745-754.

Dickerson BC, Salat DH, Bates JF, Atiya M, Killiany RJ, Greve DN et al (2004). Medial temporal lobe function and structure in mild cognitive impairment. Ann Neurol 56: 27-35.

Dickerson BC, Salat DH, Greve DN, Chua EF, Rand-Giovannetti E, Rentz DM et al (2005). Increased hippocampal activation in mild cognitive impairment compared to normal aging and $\mathrm{AD}$. Neurology 65: 404-411.

El Bahh B, Balosso S, Hamilton T, Herzog H, Beck-Sickinger AG, Sperk G et al (2005). The anti-epileptic actions of neuropeptide $\mathrm{Y}$ in the hippocampus are mediated by $\mathrm{Y} 2$ and not $\mathrm{Y} 5$ receptors. Eur J Neurosci 22: 1417-1430.

Foti S, Haberman RP, Samulski RJ, McCown TJ (2007). Adenoassociated virus-mediated expression and constitutive secretion of NPY or NPY 13-36 suppression seizure activity in vivo. Gene Therapy 14: 1534-1536.

Gallagher M, Burwell R, Burchinal M (1993). Severity of spatial learning impairment in aging: development of a learning index for performance in the Morris water maze. Behav Neurosci 107: $618-626$.

Geinisman Y, Ganeshina O, Yoshida R, Berry RW, Disterhoft JF, Gallagher M (2004). Aging, spatial learning, and total synapse number in the rat CA1 stratum radiatum. Neurobiol Aging 25: 407-416.

González-González IM, García-Tardón N, Cubelos B, Giménez C, Zafra F (2008). The glutamate transporter GLT1b interacts with the scaffold protein PSD-95. J Neurochem 105: 1834-1848.

Guzowski JF, Knierim JJ, Moser EI (2004). Ensemble dynamics of hippocampal regions CA3 and CA1. Neuron 44: 581-584.

Haberman RP, Samulski RJ, McCown TJ (2003). Attenuation of seizures and neuronal death by adeno-associated virus vector galanin expression and secretion. Nat Med 9: 1076-1080.

Hämäläinen A, Pihlajamäki M, Tanila H, Hänninen T, Niskanen E, Tervo $S$ et al (2007). Increased fMRI response during encoding in mild cognitive impairment. Neurobiol Aging 28: 1889-1903.

Hassel B, Iversen EG, Gjerstad L, Taubøll E (2001). Up-regulation of hippocampal glutamate transport during chronic treatment with sodium valproate. J Neurochem 77: 1285-1292.

Ji-qun C, Ishihara K, Nagayama T, Serikawa T, Sasa M (2005). Long-lasting antiepileptic effects of levetiracetam against epileptic seizures in the spontaneously epileptic rat (SER): differentiation of levetiracetam from conventional antiepileptic drugs. Epilepsia 46: 1362-1370.

Josselyn SA, Nguyen PV (2005). CREB, synapses and memory disorders: past progress and future challenges. Curr Drug Targets CNS Neurol Disord 4: 481-497.

Kaminski RM, Matagne A, Patsalos PN, Klitgaard H (2009). Benefit of combination therapy in epilepsy: a review of the preclinical evidence with levetiracetam. Epilepsia 50: 387-397.

Klitgaard H, Matagne A, Grimee R, Vanneste-Goemaere J, Margineanu DG (2003). Electrophysiological, neurochemical and regional effects of levetiracetam in the rat pilocarpine model of temporal lobe epilepsy. Seizure 12: 92-100.

Leutgeb JK, Leutgeb S, Moser MB, Moser EI (2007). Pattern separation in the dentate gyrus and CA3 of the hippocampus. Science 315: 961-966.

Leutgeb S, Leutgeb JK, Treves A, Moser MB, Moser EI (2004). Distinct ensemble codes in hippocampal areas CA3 and CA1. Science 305: 1295-1298.
Lister JP, Barnes CA (2009). Neurobiological changes in the hippocampus during normative aging. Arch Neurol 66: 829-833. McQuiston AR, Colmers WF (1996). Neuropeptide Y2 receptors inhibit the frequency of spontaneous but not miniature EPSCs in CA3 pyramidal cells of rat hippocampus. J Neurophysiol 76: 3159-3168.

Miller SL, Celone K, DePeau K, Diamond E, Dickerson BC, Rentz D et al (2008a). Age-related memory impairment associated with loss of parietal deactivation but preserved hippocampal activation. Proc Natl Acad Sci USA 105: 2181-2186.

Miller SL, Fenstermacher E, Bates J, Blacker D, Sperling RA, Dickerson BC (2008b). Hippocampal activation in adults with mild cognitive impairment predicts subsequent cognitive decline. J Neurol Neurosurg Psychiatry 79: 630-635.

Morris MJ, Gannan E, Stroud LM, Beck-Sickinger AG, O’Brien TJ (2007). Neuropeptide Y suppresses absence seizures in a genetic rat model primarily through effects on $\mathrm{Y}_{2}$ receptors. Eur $\mathrm{J}$ Neurosci 25: 1136-1143.

Rapp PR, Deroche PS, Mao Y, Burwell RD (2002). Neuron number in the parahippocampal region in preserved in aged rats with spatial learning deficits. Cereb Cortex 12: 1171-1179.

Rapp PR, Gallagher M (1996). Preserved neuron number in the hippocampus of aged rats with spatial learning deficits. Proc Natl Acad Sci USA 93: 9926-9930.

Perucca E (2002). Pharmacological and therapeutic properties of valproate: a summary after 35 years of clinical experience. CNS Drugs 16: 695-714.

Robitsek RJ, Fortin NJ, Koh MT, Gallagher M, Eichenbaum H (2008). Cognitive aging: a common decline of episodic recollection and spatial memory in rats. J Neurosci 28: 8945-8954.

Rose GM, Hopper A, De Vivo M, Tehim A (2005). Phosphodiesterase inhibitors for cognitive enhancement. Curr Pharm Des 11: 3329-3334.

Silva AP, Xapelli S, Pinheiro PS, Ferreira R, Lourenço J, Cristóvão $A$ et al (2005). Up-regulation of neuropeptide $\mathrm{Y}$ levels and modulation of glutamate release through neuropeptide $\mathrm{Y}$ receptors in the hippocampus of kainite-induced epileptic rats. J Neurochem 93: 163-170.

Smith TD, Adams MM, Gallagher M, Morrison JH, Rapp PR (2000). Circuit-specific alterations in hippocampal synaptophysin immunoreactivity predict spatial learning impairment in aged rats. J Neurosci 20: 6587-6593.

Steele RJ, Morris RG (1999). Delay-dependent impairment of a matching-to-place task with chronic and intrahippocampal infusion of the NMDA-antagonist D-AP5. Hippocampus 9: 118-136.

Stout SC, Owens MJ, Lindsey KP, Knight DL, Nemeroff CB (2001). Effects of sodium valproate on corticotropin-releasing factor systems in rat brain. Neuropsychopharmacology 24: 624-631.

Stratton SC, Large CH, Cox B, Davies G, Hagan RM (2003). Effects of lamotrigine and levetiracetam on seizure development in a rat amygdala kindling model. Epilepsy Res 53: 95-106.

Treves A, Rolls ET (1994). Computational analysis of the role of the hippocampus in memory. Hippocampus 4: 374-391.

Tulloch IF, Walter DS, Howe GM, Howe SJ (1982). The relationship between plasma concentration of valproic acid and its anticonvulsant and behavioural effects in the rat. Neuropharmacology 21: 555-562.

Vezzani A, Sperk G, Colmers WF (1999). Neuropeptide Y: emerging evidence for a functional role in seizure modulation. Trends Neurosci 22: 25-30.

Wilson IA, Gallagher M, Eichenbaum H, Tanila H (2006). Neurocognitive aging: prior memories hinder new hippocampal encoding. Trends Neurosci 29: 662-670.

Wilson IA, Ikonen S, Gallagher M, Eichenbaum H, Tanila H (2005). Age-associated alterations of hippocampal place cells are subregion specific. J Neurosci 25: 6877-6886.

Wilson IA, Ikonen S, McMahan RW, Gallagher M, Eichenbaum H, Tanila H (2003). Place cell rigidity correlates with impaired spatial learning in aged rats. Neurobiol Aging 24: 297-305. 